\title{
Bringing order to chaos: Hybrid modelling of a discontinuous chaotic system
}

DOI:

10.1109/VSS.2010.5544705

Link to publication record in Manchester Research Explorer

\section{Citation for published version (APA):}

Navarro-López, E. M., \& Barajas-Ramírez, J. G. (2010). Bringing order to chaos: Hybrid modelling of a discontinuous chaotic system. In Proceedings of the 201011 th International Workshop on Variable Structure Systems, VSS 2010/Proc. Int. Workshop Var. Struct. Syst., VSS (pp. 325-330). IEEE. https://doi.org/10.1109/VSS.2010.5544705

\section{Published in:}

Proceedings of the 201011 th International Workshop on Variable Structure Systems, VSS 2010|Proc. Int. Workshop Var. Struct. Syst., VSS

\section{Citing this paper}

Please note that where the full-text provided on Manchester Research Explorer is the Author Accepted Manuscript or Proof version this may differ from the final Published version. If citing, it is advised that you check and use the publisher's definitive version.

\section{General rights}

Copyright and moral rights for the publications made accessible in the Research Explorer are retained by the authors and/or other copyright owners and it is a condition of accessing publications that users recognise and abide by the legal requirements associated with these rights.

\section{Takedown policy}

If you believe that this document breaches copyright please refer to the University of Manchester's Takedown Procedures [http://man.ac.uk/04Y6Bo] or contact uml.scholarlycommunications@manchester.ac.uk providing relevant details, so we can investigate your claim.

\section{OPEN ACCESS}




\title{
Bringing Order to Chaos: Hybrid Modelling of a Discontinuous Chaotic System
}

\author{
Eva M. Navarro-López Member, IEEE, and Juan G. Barajas-Ramírez
}

\begin{abstract}
We propose a computational-oriented perspective within the study of discontinuous chaotic systems, and provide insights into the modelling, control and simulation of chaotic systems with switching dynamics. In particular, the Lorenz system in its piecewise-linear version is studied. This system is reinterpreted within the hybrid-automaton framework, and what is referred to as the Lorenz hybrid automaton is established. Furthermore, a discontinuous control which eliminates the chaotic behaviour and steers the trajectories to a desired equilibrium is proposed. An integral characteristic of the modelling framework is that the controlled system, exhibiting three discontinuity surfaces, is reduced to the composition of several Lorenz hybrid automata. The approach proposed here is especially useful in order to specify the transitions between the different system operation modes, which becomes a crucial problem due to the existence of multiple switching surfaces.
\end{abstract}

\section{MOTIVATION}

What is the best model to describe variable structure systems with multiple switching elements and exhibiting several types of complex behaviours? How to specify all their transitions in a deterministic way to facilitate the simulation? Every mathematical model is an approximation of the real world, and is full of limitations. However, some models are better than others at describing the evolution of certain physical and engineering systems. The hybrid systems framework, in particular, the computational hybridautomaton one is very adequate for modelling and controlling complex systems [1], [2], [3].

This paper is devoted to the specification and the computational abstraction of a particular discontinuous system with chaotic behaviour: the piecewise-linear (PWL) Lorenz system. The computational framework used is that of hybrid automata [2], [3], [4], [5], [6].

There has been great success in analysing and controlling the Lorenz system and what is called the generalised Lorenz family; there is a vast literature about this topic, for example [7], [8], [9]. However, there are still open problems concerning the modelling and simulation, especially, when these systems are discontinuous [10], [11], [12]. Generally, the discontinuous or PWL version of the Lorenz system presents two discontinuity surfaces, and the problem of nonuniqueness and even non-existence of solutions has to be

EMNL thanks the Research Councils United Kingdom (Fellowship EP/E500048/1). JGBR thanks CONACYT grant no. FOMIX-SLP 86513.

E.M. Navarro-López is with the School of Computer Science, The University of Manchester, Oxford Road, M13 9PL Manchester, United Kingdom (eva.navarro@cs.man.ac.uk)

J.G. Barajas-Ramírez is with Instituto Potosino de Investigación Científica y Tecnológica (IPICYT), División de Matemáticas Aplicadas, Camino a la Presa San José 2055, 78216 San Luis Potosí, SLP, México (jgbarajaseipicyt.edu.mx) faced when the trajectories either cross or slide on the discontinuity surfaces. Furthermore, due to the coexistence of several discontinuity surfaces, the problem of specification of the transitions between the system operation modes is critical, and special care has to be taken in the numerical integration [13], [14], [15]. The use of a hybrid automaton to describe the system is presented here as a solution to this problem.

This paper is inspired by Navarro's previous results related to the modelling and discrete abstraction of discontinuous dynamical systems (DDSs) under the hybrid-automaton framework [4], [5], [6].

The Lorenz hybrid automaton with 6 discrete locations is given. To prove the efficacy of the hybrid-automaton framework, a second hybrid automaton with 18 locations is proposed. This second automaton corresponds to the controlled PWL Lorenz system with three discontinuity surfaces, which is obtained by considering a sliding-modebased control. The control strategy is based on inserting a discontinuity surface on which the system presents the desired behaviour and forces the system trajectories to slide on this surface. The controlled Lorenz hybrid automaton, by following the computer science divide-and-conquer principle, is obtained by means of the composition of three Lorenz hybrid automata. Although the discontinuous Lorenz system can be effectively stabilised with smooth or even linear controllers, we propose a discontinuous-type controller in order to include within the system a third discontinuity surface, and show the potential of the framework to model and analyse discontinuous systems with multiple switching elements.

The analysis and control of discontinuous chaotic systems is not new, and has been solved in different ways [16], [17], [18], [19]. The novel contribution of this paper is the reinterpretation of these systems as hybrid automata, which leads to an alternative modelling and simulation framework. Whilst a particular example is used, the proposed approach is applicable to a broader class of systems and provides new insights into the modelling, analysis and control of discontinuous chaotic systems.

\section{THE DISCONTINUOUS CHAOTIC SYSTEM}

The starting point of our analysis is the smooth Lorenz system with the following form [20]:

$$
\begin{aligned}
& \dot{x}_{1}=a\left(x_{2}-x_{1}\right), \\
& \dot{x}_{2}=x_{1}\left(\rho-x_{3}\right)-x_{2}, \\
& \dot{x}_{3}=x_{2} x_{1}-b x_{3} .
\end{aligned}
$$




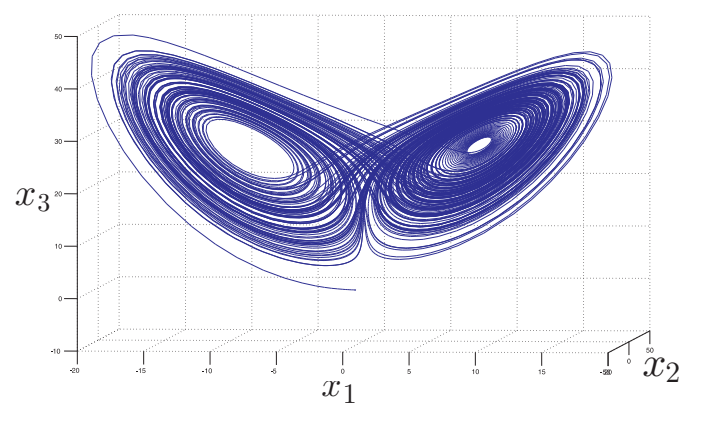

Fig. 1. Lorenz chaotic attractor.

The system state vector is defined as $x=\left(x_{1}, x_{2}, x_{3}\right)^{\mathrm{T}} \in \mathbb{R}^{3}$, and $a=10, b=\frac{8}{3}$ and $\rho=28$. It is well-known that this system for these parameters exhibits a chaotic attractor, which is shown in Fig. 1

The chaotic behaviour of the Lorenz system can be reproduced by a discontinuous version of (1), which is referred to as piecewise-linear (PWL) Lorenz system and has the form: [10], [11], [12]:

$$
\begin{aligned}
& \dot{x}_{1}=a\left(x_{2}-x_{1}\right), \\
& \dot{x}_{2}=\operatorname{sign}\left(x_{1}\right)\left(\rho-x_{3}\right)-q x_{2}, \\
& \dot{x}_{3}=\operatorname{sign}\left(x_{2}\right) x_{1}-b x_{3},
\end{aligned}
$$

with $a=1.2, b=0.15, \rho=7.0, q=0.1$. For these parameters, the PWL Lorenz system exhibits chaotic behaviour.

Let define two scalar functions $s_{1}=x_{1}$ and $s_{2}=x_{2}$. System (2) has two discontinuity surfaces:

$S_{1}:=\left\{\mathbf{x} \in \mathbb{R}^{3}: s_{1}=x_{1}=0\right\}, S_{2}:=\left\{\mathbf{x} \in \mathbb{R}^{3}: s_{2}=x_{2}=0\right\}$.

$S_{1}$ and $S_{2}$ can intersect. Now, the equivalent dynamics on $S_{1}$ and $S_{2}$ will be derived. Utkin's equivalent control method [21] will be used for this purpose, but additional considerations have to be taken into account due to the fact that on either $S_{1}$ or $S_{2}$, a second-order sliding mode appears [22], [23], [24]. By derivating twice $s_{1}$ and by considering $s_{1}=\dot{s}_{1}=\ddot{s}_{1}=0$, the relations $\operatorname{sign}\left(x_{1}\right)=q x_{2} /\left(\rho-x_{3}\right)$ and $x_{2}=0$ are obtained. Notice that $\operatorname{sign}\left(x_{1}\right)$ plays the role of the equivalent control. Consequently, it is obtained that $\operatorname{sign}\left(x_{1}\right)=0$. Following the same procedure for $s_{2}$, that is, by derivating twice $s_{2}$ and by considering $s_{2}=\dot{s}_{2}=\ddot{s}_{2}=0$, the relations $\operatorname{sign}\left(x_{2}\right)=b x_{3} / x_{1}$ and $x_{3}=\rho$ are obtained. To sum up, we have established that the equivalent dynamics on $S_{1}$ and $S_{2}$ have the form:

$$
\dot{\mathbf{x}}=\mathbf{f}_{s_{1}}(\mathbf{x})=\left(\begin{array}{c}
0 \\
0 \\
-b x_{3}
\end{array}\right), \quad \dot{\mathbf{x}}=\mathbf{f}_{s_{2}}(\mathbf{x})=\left(\begin{array}{c}
-a x_{1} \\
0 \\
0
\end{array}\right) .
$$

We again remark that for the case $x_{1}=x_{2}=0$, the dynamics $\dot{\mathbf{x}}=f_{\mathrm{s}_{1}}(\mathbf{x})$ will be considered.

We will specify and simulate system (2) by means of a hybrid automaton in order to reproduce the Lorenz chaotic attractor for the PWL Lorenz system. In addition, a discontinuous control is proposed in order to eliminate this chaotic behaviour and drive the system trajectories to the origin.

\section{THE PWL LORENZ SYSTEM AS A HYBRID AUTOMATON}

System (2) can be represented as a hybrid automaton, which will be referred to as the Lorenz hybrid automaton $\left(H_{\mathrm{L}}\right)$. The proposal of $H_{\mathrm{L}}$ is inspired by the DDS hybrid automaton - that is, a special hybrid automaton for describing discontinuous dynamical systems (DDSs) - proposed by Navarro-López in [4], [5], [6]. The following general hybrid automaton with inputs and outputs, which was also proposed in [4], [5], [6], will be used as the basic model.

Definition 1. A hybrid automaton with inputs and outputs is a collection

$$
H=(Q, E, \mathscr{X}, \Sigma, \mathscr{U}, O, \mathscr{Y}, \text { Dom, } \mathscr{F}, \text { Init }, G, R, h, r)
$$

where:

- $Q=\left\{q_{1}, q_{2}, \ldots, q_{\mathrm{N}}\right\}$ is a finite set of discrete locations. - $\mathscr{X} \subseteq \mathbb{R}^{\mathrm{n}}, \mathscr{U} \subseteq \mathbb{R}^{\mathrm{m}}$ and $\mathscr{Y} \subseteq \mathbb{R}^{\mathrm{m}}$ are the state, input and output continuous spaces, respectively. The state of $H$ is $(q, \mathbf{x}) \in Q \times \mathscr{X}$.

- $E \subseteq Q \times Q$ is a finite set of edges called transitions.

- $\Sigma=\left\{\sigma_{1}, \sigma_{2}, \ldots, \sigma_{\mathrm{M}}\right\}$ is a finite set of symbols labelling the transitions and representing the discrete input events.

- $O=\left\{o_{1}, o_{2}, \ldots, o_{\mathrm{K}}\right\}$ is a finite set of symbols representing the discrete output events.

- Dom: $Q \rightarrow 2^{\mathscr{X} \times \mathscr{U}}$ is the location domain. Dom assigns a set of continuous states and inputs to each discrete state $q_{\mathrm{i}} \in Q$, thus, $\operatorname{Dom}\left(q_{\mathrm{i}}\right) \subset \mathscr{X} \times \mathscr{U}$. As long as the system is within location $q_{\mathrm{i}}$, the continuous state $\mathbf{x}$ must satisfy $\mathbf{x} \in \operatorname{Dom}\left(q_{\mathrm{i}}\right)$.

- $\mathscr{F}=\left\{\mathbf{f}_{\mathrm{q}_{\mathrm{i}}}(\mathbf{x}, \mathbf{u}): \quad q_{\mathrm{i}} \in Q\right\}$ is the collection of vector fields describing the continuous dynamics such that $f_{\mathrm{q}_{\mathrm{i}}}: \mathscr{X} \times \mathscr{U} \rightarrow \mathscr{X}$. Each $\mathbf{f}_{\mathrm{q}_{\mathrm{i}}}(\mathbf{x}, \cdot)$ is assumed to be Lipschitz continuous on the location domain for $q_{\mathrm{i}}$ in order to ensure that the solution within $\operatorname{Dom}\left(q_{\mathrm{i}}\right)$ exists and is unique.

- Init $\subseteq Q \times \mathscr{X}$ is a set of initial states.

- $G: E \rightarrow 2^{\mathscr{X}}$ is a guard set. Funtion $G$ assigns to each transition $e=\left(q_{\mathrm{i}}, q_{\mathrm{j}}\right) \in E$ a set of continuous states $(G(e) \subset \mathscr{X})$. Each guard enables the change of discrete location.

- $R: E \times \mathscr{X} \times \mathscr{U} \rightarrow 2^{\mathscr{X}}$ is a reset map for the continuous states for each transition. It is assumed that $\forall e \in E$, $G(e) \neq \emptyset$ and $\forall \mathbf{x} \in G(e), R(e, \mathbf{x}, \mathbf{u}) \neq \emptyset$.

- $h: Q \times \mathscr{X} \times \mathscr{U} \rightarrow \mathscr{Y}$ is the continuous output mapping, there is one for each location.

- $r: Q \times \mathscr{X} \times \Sigma \times \mathscr{U} \rightarrow O$ is the discrete output map, there is one for each location.

In order to define the transitions of $H_{\mathrm{L}}$, two properties are considered:

- $x_{1}$ and $x_{2}$ cannot change sign without crossing the discontinuity surface. That is, $x_{1}$ (or $x_{2}$ ) cannot change from $x_{1}>0$ to $x_{1}<0$ directly, it has to visit first the discrete location in which $x_{1}=0$.

- Due to numerical problems for detecting the zero crossing, a zero band is considered, and instead of having $x_{1}=0, x_{1}>0$ and $x_{1}<0,\left|x_{1}\right| \leq \delta, x_{1}>\delta$ and $x_{1}<-\delta$ 
are considered, respectively, with $\delta=10^{-6}$. The same consideration is for $x_{2}$.

Definition 2. The Lorenz hybrid automaton with 6 discrete states $\left(H_{\mathrm{L}}\right)$ describing the dynamics of system (2) is a particular case of $H$ with,

- $Q=\left\{q_{1}, q_{2}, q_{3}, q_{4}, q_{5}, q_{6}\right\}, \mathscr{X} \subseteq \mathbb{R}^{3}$.

- $E=\left\{\left(q_{1}, q_{3}\right),\left(q_{1}, q_{4}\right),\left(q_{2}, q_{3}\right),\left(q_{2}, q_{4}\right),\left(q_{3}, q_{1}\right)\right.$, $\left(q_{3}, q_{2}\right),\left(q_{3}, q_{4}\right),\left(q_{3}, q_{5}\right),\left(q_{3}, q_{6}\right),\left(q_{4}, q_{1}\right),\left(q_{4}, q_{2}\right)$, $\left(q_{4}, q_{3}\right),\left(q_{4}, q_{5}\right),\left(q_{4}, q_{6}\right),\left(q_{5}, q_{3}\right),\left(q_{5}, q_{4}\right),\left(q_{6}, q_{3}\right)$, $\left.\left(q_{6}, q_{4}\right)\right\}$.

- $\Sigma=\{a, b, c, d, e, f, g, h, i\} . \Sigma=O$. One edge label is assigned to each type of guard.

- $\operatorname{Dom}\left(q_{1}\right)=\left\{\mathbf{x} \in \mathbb{R}^{3}: x_{1}>\delta, x_{2}>\delta\right\}, \operatorname{Dom}\left(q_{2}\right)=\{\mathbf{x} \in$ $\left.\mathbb{R}^{3}: x_{1}>\delta, x_{2}<-\delta\right\}, \operatorname{Dom}\left(q_{3}\right)=\left\{\mathbf{x} \in \mathbb{R}^{3}:\left|x_{1}\right|>\right.$ $\left.\delta,\left|x_{2}\right| \leq \delta\right\}, \operatorname{Dom}\left(q_{4}\right)=\left\{\mathbf{x} \in \mathbb{R}^{3}:\left|x_{1}\right| \leq \delta\right\}, \operatorname{Dom}\left(q_{5}\right)=$ $\left\{\mathbf{x} \in \mathbb{R}^{3}: x_{1}<-\delta, x_{2}>\delta\right\}, \operatorname{Dom}\left(q_{6}\right)=\left\{\mathbf{x} \in \mathbb{R}^{3}: x_{1}<\right.$ $\left.-\delta, x_{2}<-\delta\right\}$.

- Dynamics for each discrete location:

$$
\begin{aligned}
& \mathbf{f}_{q_{1}}=\left(\begin{array}{c}
a\left(x_{2}-x_{1}\right) \\
\rho-x_{3}-q x_{2} \\
x_{1}-b x_{3}
\end{array}\right), \mathbf{f}_{q_{2}}=\left(\begin{array}{c}
a\left(x_{2}-x_{1}\right) \\
\rho-x_{3}-q x_{2} \\
-x_{1}-b x_{3}
\end{array}\right), \\
& \mathbf{f}_{q_{3}}=\left(\begin{array}{c}
-a x_{1} \\
0 \\
0
\end{array}\right), \mathbf{f}_{q_{4}}=\left(\begin{array}{c}
0 \\
0 \\
-b x_{3}
\end{array}\right), \\
& \mathbf{f}_{q_{5}}=\left(\begin{array}{c}
a\left(x_{2}-x_{1}\right) \\
x_{3}-q x_{2}-\rho \\
x_{1}-b x_{3}
\end{array}\right), \mathbf{f}_{q_{6}}=\left(\begin{array}{c}
a\left(x_{2}-x_{1}\right) \\
x_{3}-q x_{2}-\rho \\
-x_{1}-b x_{3}
\end{array}\right) .
\end{aligned}
$$

The case $x_{1}=x_{2}=0$ is considered in location $q_{4}$.

- $G\left(q_{3}, q_{1}\right)=G\left(q_{4}, q_{1}\right)=\operatorname{Dom}\left(q_{1}\right), \quad G\left(q_{1}, q_{4}\right)=$ $G\left(q_{5}, q_{4}\right)=\left\{\mathbf{x} \in \mathbb{R}^{3}:\left|x_{1}\right| \leq \delta, x_{2}>\delta\right\}, G\left(q_{3}, q_{5}\right)=$ $G\left(q_{4}, q_{5}\right)=\operatorname{Dom}\left(q_{5}\right), \quad G\left(q_{1}, q_{3}\right)=G\left(q_{2}, q_{3}\right)=\{\mathbf{x} \in$ $\left.\mathbb{R}^{3}:\left|x_{2}\right| \leq \delta, x_{1}>\delta\right\}, G\left(q_{3}, q_{4}\right)=\left\{\mathbf{x} \in \mathbb{R}^{3}:\left|x_{1}\right| \leq\right.$ $\left.\delta,\left|x_{2}\right| \leq \delta\right\}, G\left(q_{5}, q_{3}\right)=G\left(q_{6}, q_{3}\right)=\left\{\mathbf{x} \in \mathbb{R}^{3}:\left|x_{2}\right| \leq\right.$ $\left.\delta, x_{1}<-\delta\right\}, \quad G\left(q_{3}, q_{2}\right)=G\left(q_{4}, q_{2}\right)=\operatorname{Dom}\left(q_{2}\right)$, $G\left(q_{2}, q_{4}\right)=G\left(q_{6}, q_{4}\right)=\left\{\mathbf{x} \in \mathbb{R}^{3}:\left|x_{1}\right| \leq \delta, x_{2}<-\delta\right\}$, $G\left(q_{3}, q_{6}\right)=G\left(q_{4}, q_{6}\right)=\operatorname{Dom}\left(q_{6}\right), G\left(q_{4}, q_{3}\right)=\operatorname{Dom}\left(q_{3}\right)$.

- $R\left(q_{\mathrm{i}}, q_{\mathrm{j}}, \mathbf{x}\right)=\{\mathbf{x}\}, \forall i, j \in\{1, \ldots, 6\}$, and $i \neq j$.

- $y=h\left(q_{\mathrm{i}}, x\right)=\mathbf{x}^{\mathrm{T}}, \forall i \in\{1, \ldots, 6\}$.

The graphical representation of the Lorenz hybrid automaton is given in Fig. 2. This hybrid automaton was simulated for $q_{0} \times \mathbf{x}_{0}=q_{6} \times\left(-2.1 \cdot 10^{-4},-5.20625 \cdot 10^{-3}, 6.895783\right)^{\mathrm{T}}$ by using the tool Ptolemy II under the environment HyVisual [25], [26]. The resulting chaotic attractor of $H_{\mathrm{L}}$ and the discrete locations visited in order to generate it are shown in Fig. 3. From the figure, as it was expected, it can be appreciated that the hybrid trajectory crosses either $S_{1}$ or $S_{2}$ without sliding on them.

\section{CONTROLLING THE HYBRID SYSTEM}

The control goal is to eliminate the chaotic behaviour and steer the trajectories of the system to the equilibrium $\mathbf{x}=$ $(0,0,0)^{\mathrm{T}}$. For this purpose, a control variable $u$ in included in system (2), having now $\dot{x}_{1}=a\left(x_{2}-x_{1}\right)+u$.

It is well-established that the most appropriate types of controllers in order to stabilise a discontinuous system is a discontinuous or switched-type controller [27]. We will

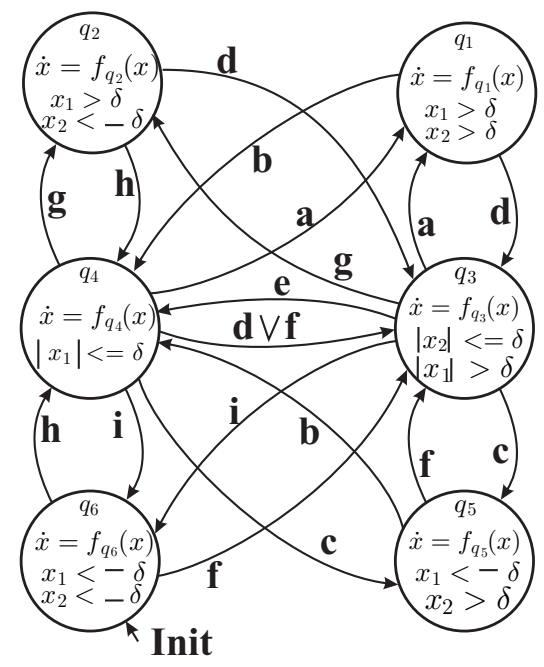

Fig. 2. Hybrid automaton $H_{\mathrm{L}}$ for system (2).
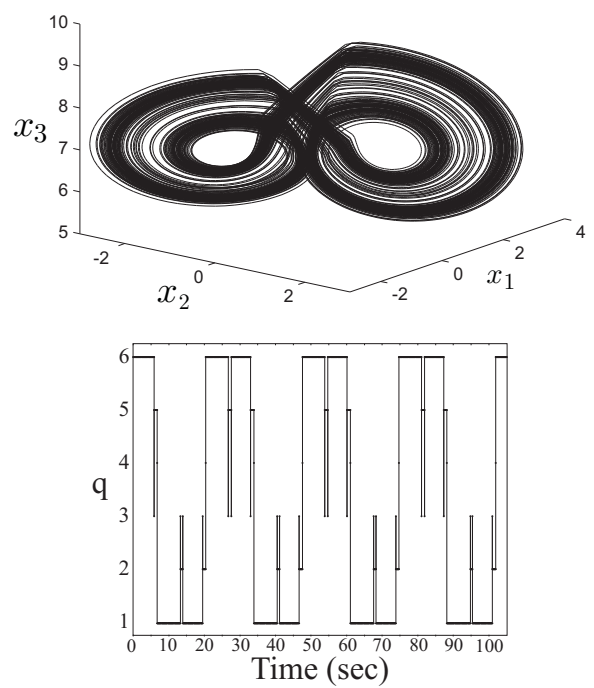

Fig. 3. Chaotic attractor of the Lorenz hybrid automaton $H_{\mathrm{L}}$ with the visited discrete locations. The chaotic attractor is obtained with a simulation time of $1800 \mathrm{secs}$, and the discrete locations are shown for the first $100 \mathrm{secs}$.

follow this recommendation and propose a discontinuous controller. Indeed, the use of a discontinuous controller which implies the inclusion of another discontinuity surface in the system - will show how powerful the hybridautomaton framework is in order to specify the multiple transitions of complex systems. Furthermore, the control scheme proposed can be useful for controlling chaotic discontinuous systems with similar structure to the Lorenz system.

\section{A. Sliding Mode-based Control}

The control goal will be achieved by inserting an attractive surface of discontinuity, $s_{\mathrm{d}}=0$, along which the system trajectories enter a sliding regime and $x_{1} \rightarrow x_{2} \rightarrow x_{3} \rightarrow 0$. 
A discontinuous control $u$ is proposed, which is inspired by the control structure proposed in [28].

We define the following scalar function:

$$
\begin{aligned}
s_{d}= & \left(x_{1}+x_{2}+x_{3}\right)+\lambda \int_{0}^{t}\left(x_{1}(\tau)-x_{3}(\tau)\right) d \tau+ \\
& +\lambda \int_{0}^{t}\left(x_{2}(\tau)-x_{3}(\tau)\right) d \tau= \\
= & \left(x_{1}+x_{2}+x_{3}\right)+\lambda\left(x_{4}+x_{5}\right), 0<\lambda<1, \\
\dot{x}_{4}= & x_{1}-x_{3}, \dot{x}_{5}=x_{2}-x_{3} .
\end{aligned}
$$

For $s_{\mathrm{d}}$ to become zero and make $s_{\mathrm{d}}=0$ be attractive (that is, to have $\left.s_{\mathrm{d}} \dot{s}_{\mathrm{d}}<0\right), \dot{s}_{\mathrm{d}}$ is forced to be:

$$
\dot{s}_{\mathrm{d}}=-\eta \operatorname{sign}\left(s_{\mathrm{d}}\right),
$$

with $\eta>0$, ensuring to have a sliding motion on $s_{\mathrm{d}}=0$ [28]. From (4) and (5), the following control $u$ is obtained:

$$
\begin{aligned}
u= & -a\left(x_{2}-x_{1}\right)+q x_{2}+b x_{3}-\lambda\left(x_{1}+x_{2}-2 x_{3}\right)- \\
& -\operatorname{sign}\left(x_{1}\right)\left(\rho-x_{3}\right)-\operatorname{sign}\left(x_{2}\right) x_{1}-\eta \operatorname{sign}\left(s_{\mathrm{d}}\right) .
\end{aligned}
$$

By applying (6) to dynamics (2), this system is obtained:

$$
\begin{aligned}
\dot{x}_{1}= & q x_{2}+b x_{3}-\lambda\left(x_{1}+x_{2}-2 x_{3}\right)- \\
& -\operatorname{sign}\left(x_{1}\right)\left(\rho-x_{3}\right)-\operatorname{sign}\left(x_{2}\right) x_{1}-\eta \operatorname{sign}\left(s_{\mathrm{d}}\right), \\
\dot{x}_{2}= & \operatorname{sign}\left(x_{1}\right)\left(\rho-x_{3}\right)-q x_{2}, \\
\dot{x}_{3}= & \operatorname{sign}\left(x_{2}\right) x_{1}-b x_{3} .
\end{aligned}
$$

The dynamics of system (7) on the surface of discontinuity $s_{\mathrm{d}}=0$ can be obtained by means of Utkin's equivalent control method [21], and have the form:

$$
\begin{aligned}
\dot{x}_{1}= & q x_{2}+b x_{3}-\lambda\left(x_{1}+x_{2}-2 x_{3}\right)- \\
& -\operatorname{sign}\left(x_{1}\right)\left(\rho-x_{3}\right)-\operatorname{sign}\left(x_{2}\right) x_{1}, \\
\dot{x}_{2}= & \operatorname{sign}\left(x_{1}\right)\left(\rho-x_{3}\right)-q x_{2}, \\
\dot{x}_{3}= & \operatorname{sign}\left(x_{2}\right) x_{1}-b x_{3} .
\end{aligned}
$$

The equivalent dynamics on the surfaces $S_{1}$ and $S_{2}$ are computed by applying Utkin's equivalent control method and following the same procedure as in Section II. The difference now is that the second-order sliding motion appears on $S_{2}$.

\section{B. Hybrid Automaton of the Controlled System}

The hybrid automaton which describes the controlled system (7) will be referred to as $H_{\mathrm{LC}}$. It has the following key characteristics:

- $H_{\mathrm{LC}}$ is obtained as the composition of three hybrid automata $H_{\mathrm{L}}$. Each of these three hybrid automata corresponds to: $s_{\mathrm{d}}=0$ (discrete locations $q_{1}-q_{6}$ ), $s_{\mathrm{d}}<0$ (discrete locations $q_{7}-q_{12}$ ) and $s_{\mathrm{d}}>0$ (discrete locations $\left.q_{13}-q_{18}\right)$.

- The surface $s_{\mathrm{d}}=0$ is attractive for all $\mathbf{x}$ and all the trajectories reach it in a finite time. Furthermore, once the trajectory reaches $s_{\mathrm{d}}=0$, it remains there. Consequently, once the system reaches locations $q_{1}-q_{6}$, its future transitions will be restricted to these six locations. This can be considered as a desired recurrent loop. In addition, we also consider as unfeasiable transitions, the ones going across $s_{\mathrm{d}}=0$.
- For simulation purposes, a zero band in considered, and $s_{\mathrm{d}}=0, s_{\mathrm{d}}<0$ and $s_{\mathrm{d}}>0$ are rewritten as $\left|s_{\mathrm{d}}\right| \leq \delta, s_{\mathrm{d}}<$ $-\delta$ and $s_{\mathrm{d}}>\delta$, respectively, with $\delta=10^{-6}$.

- When entering $q_{4}, x_{1}$ is reset to 0 ; when entering $q_{3}, x_{2}$ is reset to 0 . When entering the rest of locations, $\mathbf{x}$ is not reset.

The graphical representation of $H_{\mathrm{LC}}$ is given in two parts, in Figures 4 and 5 .

\section{BLOCK 1}

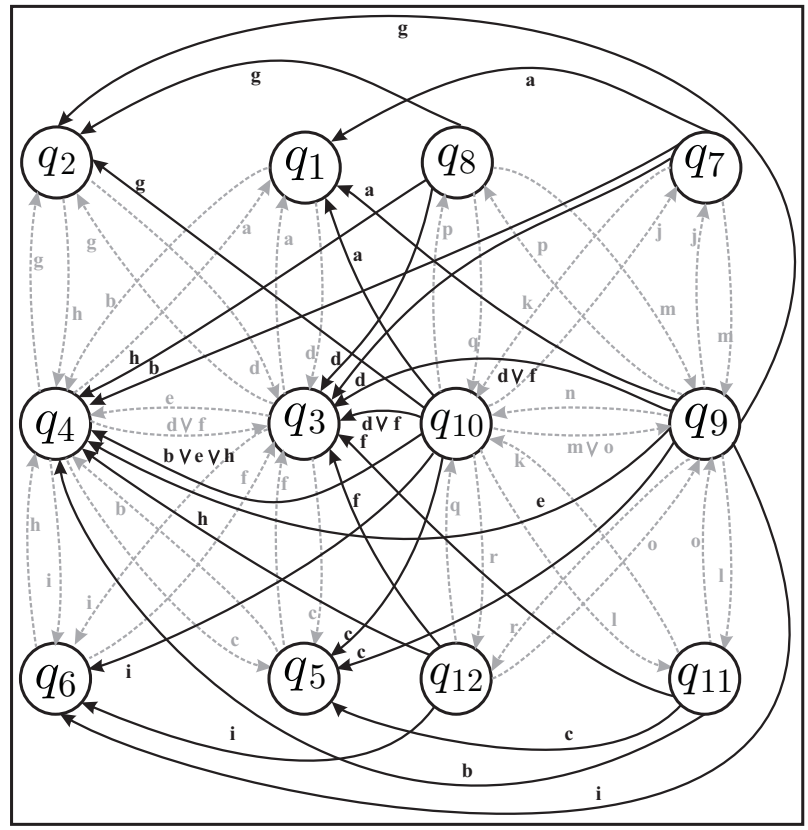

Fig. 4. Part I of the hybrid automaton for the controlled system. The dotted lines represent the transitions within each group of six locations. The solidlined transitions represent the transitions to the discontinuity surface $s_{\mathrm{d}}=0$.

The main elements of $H_{\mathrm{LC}}$ are:

\section{- Discrete locations:}

- $\operatorname{Dom}\left(q_{1}\right)=\left\{\mathbf{x} \in \mathbb{R}^{5}: x_{1}>\delta, x_{2}>\delta,\left|s_{\mathrm{d}}\right| \leq \delta\right\}$.

- $\operatorname{Dom}\left(q_{2}\right)=\left\{\mathbf{x} \in \mathbb{R}^{5}: x_{1}>\delta, x_{2}<-\delta,\left|s_{\mathrm{d}}\right| \leq \delta\right\}$.

- $\operatorname{Dom}\left(q_{3}\right)=\left\{\mathbf{x} \in \mathbb{R}^{5}:\left|x_{1}\right|>\delta,\left|x_{2}\right| \leq \delta,\left|s_{\mathrm{d}}\right| \leq \delta\right\}$.

- $\operatorname{Dom}\left(q_{4}\right)=\left\{\mathbf{x} \in \mathbb{R}^{5}:\left|x_{1}\right| \leq \delta,\left|s_{\mathrm{d}}\right| \leq \delta\right\}$.

- $\operatorname{Dom}\left(q_{5}\right)=\left\{\mathbf{x} \in \mathbb{R}^{5}: x_{1}<-\delta, x_{2}>\delta,\left|s_{\mathrm{d}}\right| \leq \delta\right\}$.

- $\operatorname{Dom}\left(q_{6}\right)=\left\{\mathbf{x} \in \mathbb{R}^{5}: x_{1}<-\delta, x_{2}<-\delta,\left|s_{\mathrm{d}}\right| \leq \delta\right\}$.

- $\operatorname{Dom}\left(q_{7}\right)=\left\{\mathbf{x} \in \mathbb{R}^{5}: x_{1}>\delta, x_{2}>\delta, s_{\mathrm{d}}<-\delta\right\}$.

- $\operatorname{Dom}\left(q_{8}\right)=\left\{\mathbf{x} \in \mathbb{R}^{5}: x_{1}>\delta, x_{2}<-\delta, s_{\mathrm{d}}<-\delta\right\}$.

- $\operatorname{Dom}\left(q_{9}\right)=\left\{\mathbf{x} \in \mathbb{R}^{5}:\left|x_{1}\right|>\delta,\left|x_{2}\right| \leq \delta, s_{\mathrm{d}}<-\delta\right\}$.

- $\operatorname{Dom}\left(q_{10}\right)=\left\{\mathbf{x} \in \mathbb{R}^{5}:\left|x_{1}\right| \leq \delta, s_{\mathrm{d}}<-\delta\right\}$.

- $\operatorname{Dom}\left(q_{11}\right)=\left\{\mathbf{x} \in \mathbb{R}^{5}: x_{1}<-\delta, x_{2}>\delta, s_{\mathrm{d}}<-\delta\right\}$.

- $\operatorname{Dom}\left(q_{12}\right)=\left\{\mathbf{x} \in \mathbb{R}^{5}: x_{1}<-\delta, x_{2}<-\delta, s_{\mathrm{d}}<\right.$ $-\delta\}$.

- $\operatorname{Dom}\left(q_{13}\right)=\left\{\mathbf{x} \in \mathbb{R}^{5}: x_{1}>\delta, x_{2}>\delta, s_{\mathrm{d}}>\delta\right\}$.

- $\operatorname{Dom}\left(q_{14}\right)=\left\{\mathbf{x} \in \mathbb{R}^{5}: x_{1}>\delta, x_{2}<-\delta, s_{\mathrm{d}}>\delta\right\}$.

- $\operatorname{Dom}\left(q_{15}\right)=\left\{\mathbf{x} \in \mathbb{R}^{5}:\left|x_{1}\right|>\delta,\left|x_{2}\right| \leq \delta, s_{\mathrm{d}}>\delta\right\}$.

- $\operatorname{Dom}\left(q_{16}\right)=\left\{\mathbf{x} \in \mathbb{R}^{5}:\left|x_{1}\right| \leq \delta, s_{\mathrm{d}}>\delta\right\}$.

- $\operatorname{Dom}\left(q_{17}\right)=\left\{\mathbf{x} \in \mathbb{R}^{5}: x_{1}<-\delta, x_{2}>\delta, s_{\mathrm{d}}>\delta\right\}$.

- $\operatorname{Dom}\left(q_{18}\right)=\left\{\mathbf{x} \in \mathbb{R}^{5}: x_{1}<-\delta, x_{2}<-\delta, s_{\mathrm{d}}>\delta\right\}$. 
BLOCK 2

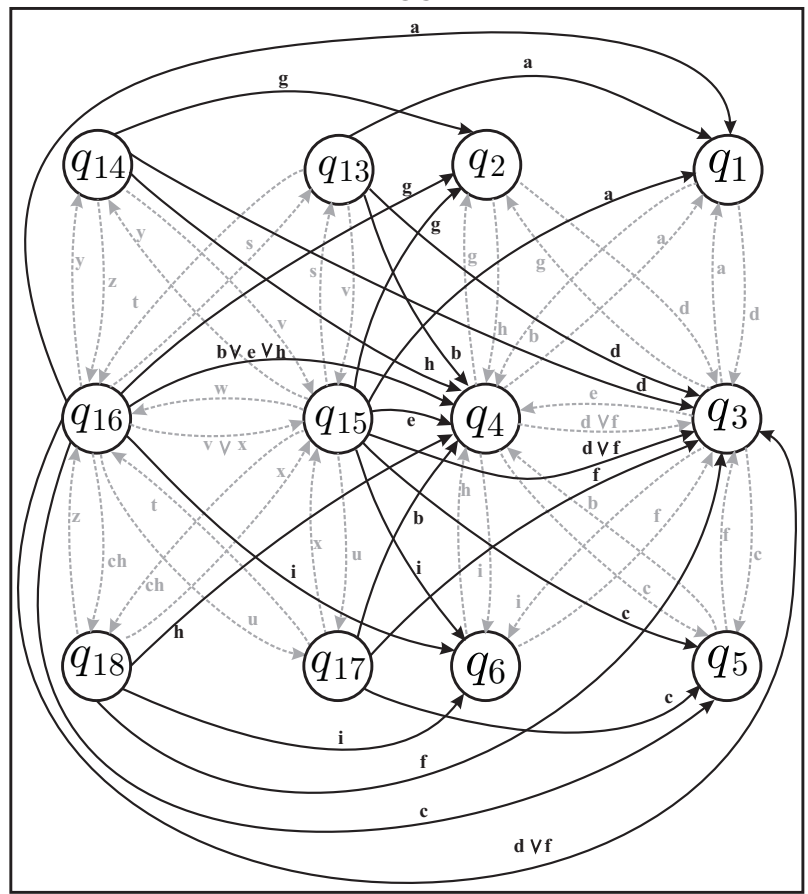

Fig. 5. Part II of the hybrid automaton for the controlled system. The dotted lines represent the transitions within each group of six locations. The solid-lined transitions represent the transitions to the discontinuity surface $s_{\mathrm{d}}=0$.

- Dynamics for each location: for each location $\dot{x}_{4}=$ $x_{1}-x_{3}, \dot{x}_{5}=x_{2}-x_{3}$ is considered, which is part of the controller dynamics, in addition to the system dynamics for $x_{1}, x_{2}$ and $x_{3}$ as follows:

$$
\begin{aligned}
& \mathbf{f}_{q_{1}}=\left(\begin{array}{c}
\varphi_{1}(\mathbf{x})-x_{1} \\
\rho-x_{3}-q x_{2} \\
x_{1}-b x_{3}
\end{array}\right), \mathbf{f}_{q_{2}}=\left(\begin{array}{c}
\varphi_{1}(\mathbf{x})+x_{1} \\
\rho-x_{3}-q x_{2} \\
-x_{1}-b x_{3}
\end{array}\right) \\
& \mathbf{f}_{q_{3}}=\left(\begin{array}{c}
-\lambda\left(x_{1}-2 \rho\right) \\
0 \\
0
\end{array}\right), \mathbf{f}_{q_{4}}=\left(\begin{array}{c}
0 \\
b x_{3}-\lambda\left(x_{2}-2 x_{3}\right) \\
-b x_{3}
\end{array}\right) \\
& \mathbf{f}_{q_{5}}=\left(\begin{array}{c}
\varphi_{2}(\mathbf{x})-x_{1} \\
x_{3}-q x_{2}-\rho \\
x_{1}-b x_{3}
\end{array}\right), \mathbf{f}_{q_{6}}=\left(\begin{array}{c}
\varphi_{2}(\mathbf{x})+x_{1} \\
x_{3}-q x_{2}-\rho \\
-x_{1}-b x_{3}
\end{array}\right) \\
& \mathbf{f}_{q_{7}}=\left(\begin{array}{c}
\varphi_{1}(\mathbf{x})-x_{1}+\eta \\
\rho-x_{3}-q x_{2} \\
x_{1}-b x_{3}
\end{array}\right), \mathbf{f}_{q_{8}}=\left(\begin{array}{c}
\varphi_{1}(\mathbf{x})+x_{1}+\eta \\
\rho-x_{3}-q x_{2} \\
-x_{1}-b x_{3}
\end{array}\right), \\
& \mathbf{f}_{q_{9}}=\left(\begin{array}{c}
-\lambda\left(x_{1}-2 \rho\right)+\eta \\
0 \\
0
\end{array}\right), \mathbf{f}_{q_{10}}=\left(\begin{array}{c}
0 \\
b x_{3}-\lambda\left(x_{2}-2 x_{3}\right) \\
-b x_{3}
\end{array}\right) \\
& \mathbf{f}_{q_{11}}=\left(\begin{array}{c}
\varphi_{2}(\mathbf{x})-x_{1}+\eta \\
x_{3}-q x_{2}-\rho \\
x_{1}-b x_{3}
\end{array}\right), \mathbf{f}_{q_{12}}=\left(\begin{array}{c}
\varphi_{2}(\mathbf{x})+x_{1}+\eta \\
x_{3}-q x_{2}-\rho \\
-x_{1}-b x_{3}
\end{array}\right) \\
& \mathbf{f}_{q_{13}}=\left(\begin{array}{c}
\varphi_{1}(\mathbf{x})-x_{1}-\eta \\
\rho-x_{3}-q x_{2} \\
x_{1}-b x_{3}
\end{array}\right), \mathbf{f}_{q_{14}}=\left(\begin{array}{c}
\varphi_{1}(\mathbf{x})+x_{1}-\eta \\
\rho-x_{3}-q x_{2} \\
-x_{1}-b x_{3}
\end{array}\right)
\end{aligned}
$$

$$
\begin{aligned}
\mathbf{f}_{q_{15}} & =\left(\begin{array}{c}
-\lambda\left(x_{1}-2 \rho\right)-\eta \\
0 \\
0
\end{array}\right), \mathbf{f}_{q_{16}}=\left(\begin{array}{c}
0 \\
b x_{3}-\lambda\left(x_{2}-2 x_{3}\right) \\
-b x_{3}
\end{array}\right) \\
\mathbf{f}_{q_{17}} & =\left(\begin{array}{c}
\varphi_{2}(\mathbf{x})-x_{1}-\eta \\
x_{3}-q x_{2}-\rho \\
x_{1}-b x_{3}
\end{array}\right), \mathbf{f}_{q_{18}}=\left(\begin{array}{c}
\varphi_{2}(\mathbf{x})+x_{1}-\eta \\
x_{3}-q x_{2}-\rho \\
-x_{1}-b x_{3}
\end{array}\right)
\end{aligned}
$$

with $\varphi_{1}(\mathbf{x})=q x_{2}+b x_{3}-\lambda\left(x_{1}+x_{2}-2 x_{3}\right)-\left(\rho-x_{3}\right)$ and $\varphi_{2}(\mathbf{x})=q x_{2}+b x_{3}-\lambda\left(x_{1}+x_{2}-2 x_{3}\right)+\rho-x_{3}$.

- Conditions for a transition (guards). The 27 symbols associated with the transitions represent the 27 types of guards in the hybrid automaton, that is:

- For $\left|s_{\mathrm{d}}\right| \leq \delta$, locations $q_{1}-q_{6}$ :

$$
\begin{aligned}
& \mathbf{a} \Leftrightarrow x_{1}>\delta, x_{2}>\delta,\left|s_{\mathrm{d}}\right| \leq \delta, \\
& \mathbf{b} \Leftrightarrow\left|x_{1}\right| \leq \delta, x_{2}>\delta,\left|s_{\mathrm{d}}\right| \leq \delta, \\
& \mathbf{c} \Leftrightarrow x_{1}<-\delta, x_{2}>\delta,\left|s_{\mathrm{d}}\right| \leq \delta, \\
& \mathbf{d} \Leftrightarrow x_{1}>\delta,\left|x_{2}\right| \leq \delta,\left|s_{\mathrm{d}}\right| \leq \delta, \\
& \mathbf{e} \Leftrightarrow\left|x_{1}\right| \leq \delta,\left|x_{2}\right| \leq \delta,\left|s_{\mathrm{d}}\right| \leq \delta, \\
& \mathbf{f} \Leftrightarrow x_{1}<-\delta,\left|x_{2}\right| \leq \delta,\left|s_{\mathrm{d}}\right| \leq \delta, \\
& \mathbf{g} \Leftrightarrow x_{1}>\delta, x_{2}<-\delta,\left|s_{\mathrm{d}}\right| \leq \delta, \\
& \mathbf{h} \Leftrightarrow\left|x_{1}\right| \leq \delta, x_{2}<-\delta,\left|s_{\mathrm{d}}\right| \leq \delta, \\
& \mathbf{i} \Leftrightarrow x_{1}<-\delta, x_{2}<-\delta,\left|s_{\mathrm{d}}\right| \leq \delta,
\end{aligned}
$$

- For $s_{\mathrm{d}}<-\delta$, locations $q_{7}-q_{12}$ :

$$
\begin{aligned}
& \mathbf{j} \Leftrightarrow x_{1}>\delta, x_{2}>\delta, s_{\mathrm{d}}<-\delta, \\
& \mathbf{k} \Leftrightarrow\left|x_{1}\right| \leq \delta, x_{2}>\delta, s_{\mathrm{d}}<-\delta, \\
& \mathbf{l} \Leftrightarrow x_{1}<-\delta, x_{2}>\delta, s_{\mathrm{d}}<-\delta, \\
& \mathbf{m} \Leftrightarrow x_{1}>\delta,\left|x_{2}\right| \leq \delta, s_{\mathrm{d}}<-\delta, \\
& \mathbf{n} \Leftrightarrow\left|x_{1}\right| \leq \delta,\left|x_{2}\right| \leq \delta, s_{\mathrm{d}}<-\delta, \\
& \mathbf{o} \Leftrightarrow x_{1}<0,\left|x_{2}\right| \leq \delta, s_{\mathrm{d}}<-\delta, \\
& \mathbf{p} \Leftrightarrow x_{1}>0, x_{2}<0, s_{\mathrm{d}}<-\delta, \\
& \mathbf{q} \Leftrightarrow\left|x_{1}\right| \leq \delta, x_{2}<0, s_{\mathrm{d}}<-\delta, \\
& \mathbf{r} \Leftrightarrow x_{1}<0, x_{2}<0, s_{\mathrm{d}}<\delta,
\end{aligned}
$$

- For $s_{\mathrm{d}}>\delta$, locations $q_{13}-q_{18}$ :

$$
\begin{aligned}
& \mathbf{s} \Leftrightarrow x_{1}>\delta, x_{2}>\delta, s_{\mathrm{d}}>\delta, \\
& \mathbf{t} \Leftrightarrow\left|x_{1}\right| \leq \delta, x_{2}>\delta, s_{\mathrm{d}}>\delta, \\
& \mathbf{u} \Leftrightarrow x_{1}<-\delta, x_{2}>\delta, s_{\mathrm{d}}>\delta, \\
& \mathbf{v} \Leftrightarrow x_{1}>\delta,\left|x_{2}\right| \leq \delta, s_{\mathrm{d}}>\delta, \\
& \mathbf{w} \Leftrightarrow\left|x_{1}\right| \leq \delta,\left|x_{2}\right| \leq \delta, s_{\mathrm{d}}>\delta, \\
& \mathbf{x} \Leftrightarrow x_{1}<-\delta,\left|x_{2}\right| \leq \delta, s_{\mathrm{d}}>\delta, \\
& \mathbf{y} \Leftrightarrow x_{1}>\delta, x_{2}<-\delta, s_{\mathrm{d}}>\delta, \\
& \mathbf{z} \Leftrightarrow\left|x_{1}\right| \leq \delta, x_{2}<-\delta, s_{\mathrm{d}}>\delta, \\
& \mathbf{c h} \Leftrightarrow x_{1}<-\delta, x_{2}<-\delta, s_{\mathrm{d}}>\delta .
\end{aligned}
$$

The hybrid automaton $H_{\mathrm{LC}}$ was simulated for the initial hybrid state $q_{0} \times \mathbf{x}_{0}=q_{18} \times\left(-2.1 \cdot 10^{-4},-5.20625\right.$. $\left.10^{-3}, 6.895783\right)^{\mathrm{T}}$, and for the controller parameters $\eta=3$, $\lambda=0.1$ and $\eta=0.3, \lambda=0.01$. The results are presented in Figures 6 and 7.

As it was expected, the final discrete location for the controlled system is location $q_{4}$, for which $x_{1}=x_{2}=x_{3}=$ 

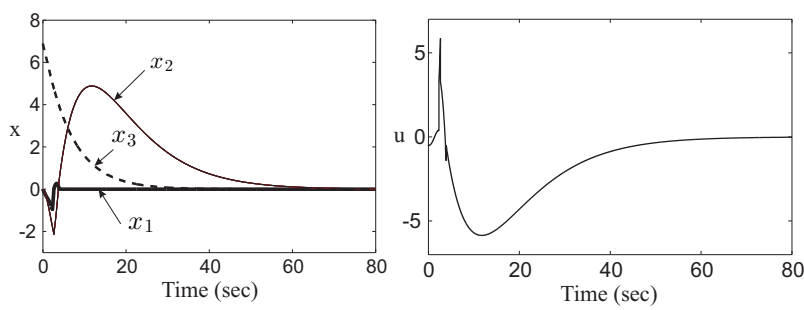

Fig. 6. Response of the controlled Lorenz hybrid automaton $H_{\mathrm{LC}}$ with $\eta=3$ and $\lambda=0.1$.
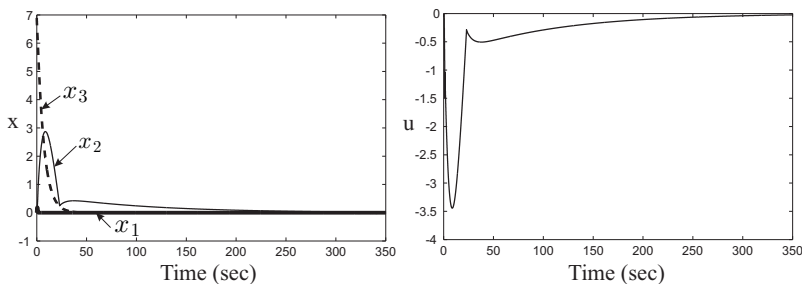

Fig. 7. Response of the controlled Lorenz hybrid automaton $H_{\mathrm{LC}}$ with $\eta=0.3$ and $\lambda=0.01$.

$s_{\mathrm{d}}=0$ is obtained. Notice that from dynamics $\dot{\mathbf{x}}=f_{\mathrm{q}_{4}}(\mathbf{x})$, the trajectories are ensured to converge to $x_{2}=x_{3}=x_{1}=0$, because $x_{1}$ is always zero entering and remaining within $q_{4}$. Changes in $\eta$ and $\lambda$ influence the time of convergence to $s_{\mathrm{d}}=0$. The bigger $\eta$ and $\lambda$ are, the faster $s_{\mathrm{d}}$ approaches zero. However, this implies a higher control effort.

A complete study of the controlled system is necessary. In particular, the analysis of the change of stability properties when some key parameters are changed (e.g., $\lambda$ ), in addition to the analysis of complex behaviours, unexpected transitions and bifurcations due to the interaction of three discontinuity surfaces in the system. This is out of the scope of this paper.

\section{CONCLUSIONS}

The Lorenz hybrid automaton and the controlled Lorenz hybrid automaton are defined in this paper in order to formulate discontinuous chaotic systems with several discontinuity surfaces within the hybrid-automaton framework. The proposed hybrid automata are especially effective in the specification of the transitions between the different modes of operation of the system, which becomes critical when multiple switching elements are present. The use of a computational framework for specifying the dynamical properties of the system can facilitate the computer-sciencebased control of chaotic systems.

\section{ACKNOWLEDGMENTS}

Thank you very much to Francisco Javier Cárdenas Flores for his support in the simulations in Ptolemy II.

\section{REFERENCES}

[1] M. Buss, M. Glocker, M. Hardt, O. von Stryk, R. Bulirsch and G. Schmidt, "Nonlinear Hybrid Dynamical Systems: Modelling, Optimal Control, and Applications", in Modelling, Analysis and Design of Hybrid Systems, LNCIS 279, Springer-Verlag, Berlin, 2002, pp 311335 .
[2] T.A. Henzinger, "The Theory of Hybrid Automata", in 11th IEEE Symposium of Logic in Computer Science, 1996, vol. 1, pp 278-292.

[3] J. Lygeros, K.H. Johansson, S.N. Simić, J. Zhang and S. Sastry, "Dynamical Properties of Hybrid Automata", IEEE Trans. on Aut. Control, vol. 48(1), 2003, pp 2-17.

[4] E.M. Navarro-López, "Hybrid Modelling of a Discontinuous Dynamical System Including Switching Control", in Second IFAC Conference on Analysis and Control of Chaotic Systems, London, UK, 2009, pp 1-6.

[5] E.M. Navarro-López,"Hybrid-automaton Models for Simulating Systems with Sliding Motion: Still a Challenge", in Third IFAC Conference on Analysis and Design of Hybrid Systems, Zaragoza, Spain, 2009, pp 322-327.

[6] E.M. Navarro-López and R. Carter, "Hybrid Automata: An Insight into the Discrete Abstraction of Discontinuous Systems", International Journal of Systems Science, 2010, under review.

[7] G. Chen and X. Dong, From Chaos to Order: Methodologies, Perspectives and Applications, World Scientific Publication Corporation, Singapore, 1998.

[8] T. Li, G. Chen and Y. Tang, "On Stability and Bifurcation of Chen's System", Chaos, Solitons \& Fractals, vol. 19, 2004, pp 1269-1282.

[9] C. Sparrow, The Lorenz Equations: Bifurcation, Chaos and Strange Attractor, Springer-Verlag, New York, 1982.

[10] E.H. Baghious and P. Jarry, "Lorenz Attractor from Differential Equations with Piecewise Linear Terms", Int. J. Bifurcation and Chaos, vol. 3(1), 1993, pp 201-210.

[11] M.A. Aziz-Alaoui and G. Chen, "Asymptotic Analysis of a New Piecewise-linear Chaotic System", Int. J. Bifurcation and Chaos, vol. 12(1), 2002, pp 147-157.

[12] J. Liu, J. Lu and X. Wu, "Bridge the Gap Between the PWL Lorenz and PWL Chens System", in 8th Int. Conference on Control, Automation Robotics and Vision, Kunming, 2004, pp 1368-1373.

[13] V. Acary and B. Brogliato, Numerical Methods for Nonsmooth Dynam ical Systems, LNACM, vol. 35, Springer-Verlag, Heidelberg, 2008.

[14] F. Zhang, M. Yeddanapudi and P.J. Mosterman, "Zero-crosing Location and Detection Algorithms for Hybrid System Simulation", in 17th IFAC Triennial World Congress, Seoul, Korea, 2008, pp 7967-7972.

[15] F. Zhao and V.I. Utkin, "Adaptive Simulation and Control Variablestructure Control Systems in Sliding Regimes", Automatica, vol. 32(7), 1996, pp 1037-1042.

[16] J. Awrejcewicz and C.H. Lamarque, Bifurcation and Chaos in Nonsmooth Mechanical Systems, World Scientific Series on Nonlinear Science, Series A, vol. 45, 2003.

[17] M.-F. Danca, "Controlling Chaos in Discontinuous Dynamical Systems", Chaos, Solitons \& Fractals, vol. 22, 2004, pp 605-612.

[18] T.C. Kuo, Y.J. Huang, C.H. Chang and C.Y. Chen, "Robust Suppression Sliding Mode Control for Uncertain Duffing-Holmes Chaotic Systems", Engineering Letters, vol. 16(3), 2008.

[19] D.I. Rosas Almeida, J. Álvarez and J.G. Barajas, "Robust Synchronization of Sprott Circuits Using Sliding Mode Control”, Chaos, Solitons \& Fractals, vol. 30, 2006, pp 11-18.

[20] S. Čelikovský and G. Chen, "On a Generalized Lorenz Canonical form of Chaotic Systems", Int. J. Bifurcation and Chaos, vol. 12(1), 2002, pp 1789-1812.

[21] V.I. Utkin, Sliding Modes in Control Optimization, Springer-Verlag, Berlin, 1992.

[22] A. Levant, "Principles of 2-Sliding Mode Design", Automatica, vol. 43, 2007, pp 576-586.

[23] G. Bartolini, A. Levant, A. Ferrara and E. Usai, "On Second-order Sliding-mode Controllers", in Lecture Notes in Control and Information Sciences, vol. 247, 1999, pp 331-350.

[24] Y.B. Shtessel, L. Fridman and A. Zinober (Eds.), "Special Issue: Advances in Higher Order Sliding Mode Control", Int. J. of Robust and Nonlinear Control, vol. 18(4-5), 2008, pp 381-585.

[25] E.A. Lee and H. Zheng, "Operational Semantics of Hybrid Systems", in Proc. of Hybrid Systems: Computation and Control, LNCS, vol. 3414, 2005, pp 25-53.

[26] E.A. Lee, Finite State Machines and Modal Models in Ptolemy II, Technical Report UCB/EECS-2009-151, University of California at Berkeley, November, 2009.

[27] D. Liberzon, Switching in Systems and Control, Birkhäuser, 2003.

[28] E.M. Navarro-López and E. Licéaga-Castro, "Non-desired Transitions and Sliding-mode Control of a Multi-DOF Mechanical System with Stick-slip Oscillations", Chaos, Solitons \& Fractals, vol. 41, 2009, pp 2035-2044. 